\title{
Endovascular management of complex superior sagittal sinus dural arteriovenous fistula
}

\author{
André Beer-Furlan, MD, Krishna C. Joshi, MBBS, MCh, Hormuzdiyar H. Dasenbrock, MD, \\ and Michael Chen, MD
}

Department of Neurological Surgery, Rush University Medical Center, Chicago, Illinois

Superior sagittal sinus (SSS) dural arteriovenous fistulas (DAVFs) are rare and present unique challenges to treatment. Complex, often bilateral, arterial supply and involvement of large volumes of eloquent cortical venous drainage may necessitate multimodality therapy such as endovascular, microsurgical, and stereotactic radiosurgery techniques. The authors present a complex SSS DAVF associated with an occluded/severely stenotic SSS. The patient underwent a successful endovascular transvenous approach with complete obliteration of the SSS. The authors discuss the management challenges faced on this case.

The video can be found here: https://youtu.be/-rztg0_cBXY.

KEYWORDS endovascular; dural arteriovenous fistula; superior sagittal sinus; transvenous; cerebral venous thrombosis; video 